\title{
SYMMETRIES AND FIRST INTEGRALS FOR NON-VARIATIONAL EQUATIONS
}

\author{
DIEGO CATALANO FERRAIOLI \\ Dipartimento di Matematica, Università di Milano \\ via Saldini 50, I-20133 Milano, Italy \\ catalano@mat.unimi.it \\ PAOLA MORANDO \\ Dipartimento di Matematica, Politecnico di Torino \\ Corso Duca degli Abruzzi 24, I-10129 Torino, Italy \\ paola.morando@polito.it
}

Received 2 July 2007

Accepted 20 July 2007

\begin{abstract}
For a class of exterior ideals, we present a method associating first integrals of the characteristic distributions to symmetries of the ideal. The method is applied, under some assumptions, to the study of first integrals of ordinary differential equations and first order partial differential equations as well as to the determination of first integrals for integrable distributions of vector fields.
\end{abstract}

Keywords: First integrals; invariants; symmetries; exterior ideals.

\section{Introduction}

In recent years an increasing attention was devoted to the search for conservation laws related to symmetries of differential equations (see, for example $[2,7,8,10,12$, $13,18]$ for a non-variational approach and $[3,16,17]$ for a variational point of view, Noether's theorem and its extensions).

The paper follows this line, using the framework of exterior ideals and their symmetries. This approach allows us to obtain a general result which can be specialized to different situations such as ordinary differential equations (ODEs), partial differential equations (PDEs) as well as distributions of vector fields. In this approach also the variational case can be considered, but our results do not give a generalization of Noether's theorem.

The main point of the paper is the possibility to associate to any infinitesimal symmetry of an exterior ideal $\mathcal{I}$, satisfying a suitable closure condition, a function that is constant along the integral manifolds of the characteristic distribution of $\mathcal{I}$.

By using this result, given a ODE $\mathcal{E}$ (satisfying suitable conditions), we associate to any symmetry of $\mathcal{E}$, a first integral of $\mathcal{E}$. More precisely, we associate to $\mathcal{E}$ the 
exterior ideal $\mathcal{I}$ generated by the contact forms restricted to $\mathcal{E}$. In this case the characteristic distribution of $\mathcal{I}$ is one dimensional and its integral lines are jet prolongations of solutions of $\mathcal{E}$. Then our result associates to any symmetry of $\mathcal{I}$ a first integral for $\mathcal{E}$. Analogous results hold for PDEs and integrable distributions of vector fields.

We point out that, in order to apply this method to different situations such as ODEs, PDEs or completely integrable distributions, the first step is to find the "good" exterior ideal $\mathcal{I}$ associated to the problem. The choice of this ideal may be not unique; for example, in the case of distributions, we exhibit two different choices for $\mathcal{I}$ sharing the same symmetries and leading to the same first integrals.

The second step is to discuss the cases satisfying the closure condition given in Lemma 1 (see below). For example, in the case of a distribution generated by a single vector field $Y$ on a manifold $M$, this condition is a generalization of $\operatorname{div}(Y)=0$. In fact, in this case, our main condition on $\mathcal{I}$ requires that $\operatorname{div}\left(e^{-K} Y\right)=0$, for a function $K$ on $M$. Then we recover and slightly generalize (for $K \neq 0$ ) a result of Crampin (see $[7,9]$ ) associating to any symmetry of a divergenceless vector field $Y$ a first integral of $Y$. Moreover, our general setting allows us to extend to an $n$-dimensional integrable distribution $(n>1)$ the result given by Crampin for a single vector field. In the general case of an exterior ideal generated by the forms $\theta_{1}, \ldots, \theta_{n} \in \Lambda^{*}(M)$, we show that the closure condition of Lemma 1 is equivalent to the existence of a function $K$ such that $e^{-K} \theta_{1} \wedge \cdots \wedge \theta_{n}$ is a closed form. Then we discuss the relations between this condition and the differential closure of the ideal, and we provide a coordinate expression for this condition for a system of ODEs.

In the case of variational ODEs we may consider the ideal $\mathcal{I}$ generated by the exterior differential of the Hamilton-Cartan 1-form, which automatically satisfies the closure condition. Then, by using our result, we associate to any symmetry of $\mathcal{I}$ a first integral of the Euler-Lagrange equations. In this sense our result is analogous of Noether's theorem, albeit the non-trivial first integrals here correspond to non Noether symmetries (see Appendix A). Any first integral obtained in this way can also be associated to a Cartan symmetry by using a generalization of Noether's theorem [16].

An outline of the paper is as follows. In Sec. 2, in order to fix notations and for the convenience of the reader, we collect some of the main definitions and propositions on exterior ideals and distributions of vector fields together with some notions from the geometric theory of differential equations. In Sec. 3 we present our main results on exterior ideals (which will be specialized in the further applications). In Sec. 4 we apply the results of Sec. 3 to distributions of vector fields and differential equations. In Sec. 5 a number of simple examples are presented in order to show that the considered class of exterior ideals is nonempty in the case of ODEs, PDEs and distributions. Finally, in Appendix A we discuss the relations between our approach and extensions of Noether's theorem in the case of ODEs of variational type. 


\section{Preliminaries}

In this section we collect some basic notions from the theory of exterior ideals and the geometric approach to differential equations. The reader is referred to $[1,4,5,11,15,20]$ for further details.

From now on $M$ will denote a smooth $n$-dimensional manifold and $\Lambda^{*}(M)$ the graded algebra of differential forms on $M$.

We say that $\mathcal{I} \subset \Lambda^{*}(M)$ is an exterior ideal (or Cartan ideal) if and only if (a) it is an ideal in $\Lambda^{*}(M)$ under exterior product, and (b) $\mathcal{I}^{k}:=\mathcal{I} \cap \Lambda^{k}(M)$ is a module over $\Lambda^{0}(M)$ for all $k=0, \ldots, n$. In other words, (a) for all $\psi \in \Lambda^{*}(M)$, $\eta \in \mathcal{I}, \psi \wedge \eta \in \mathcal{I}$; and (b) for all $f_{i} \in \Lambda^{0}(M), \beta_{i} \in \mathcal{I}^{k}(i=1,2), f_{1} \beta_{1}+f_{2} \beta_{2} \in \mathcal{I}^{k}$ (for all $k=0, \ldots, n$ ).

Let $i: S \rightarrow M$ be a smooth submanifold of $M$ (here and below $i$ is the canonical inclusion); $S$ is said to be an integral manifold of the exterior ideal $\mathcal{I}$ if and only if $i^{*}(\eta)=0$ for all $\eta \in \mathcal{I}$. In other words, $S \subset M$ is an integral manifold of $\mathcal{I}$ if and only if all $\eta \in \mathcal{I}$ vanish on $S$.

The exterior ideal $\mathcal{I}$ is said to be generated by the forms $\theta^{1}, \ldots, \theta^{h}$, which is denoted by $\mathcal{I}=\left\langle\theta^{1}, \ldots, \theta^{h}\right\rangle$, if each $\zeta \in \mathcal{I}$ can be written as $\zeta=\sum_{i} \rho_{i} \wedge \theta^{i}$ for a suitable choice of $\rho_{i} \in \Lambda^{*}(M), i=1, \ldots, h$; when $\rho_{i} \in \Lambda^{0}(M)$, by $\rho_{i} \wedge \theta^{i}$ one simply means $\rho_{i} \theta^{i}$. If $\mathcal{I}$ is generated by a collection of 1 -forms it will be called simply generated.

A simple consequence of definitions is that if $\mathcal{I}=\left\langle\theta^{1}, \ldots, \theta^{h}\right\rangle$, then $i: S \rightarrow M$ is an integral manifold for $\mathcal{I}$ if and only if $i^{*}\left(\theta^{i}\right)=0$ for all $i=1, \ldots, h$.

The exterior ideal $\mathcal{I}$ is said to be differentially closed if it is closed under exterior differentiation, i.e., if $d \eta \in \mathcal{I}$ for all $\eta \in \mathcal{I}$. In this case one can also say that $\mathcal{I}$ is a differential ideal.

In the applications usually one considers, without loss of generality, only exterior ideals which do not include 0 -forms. In fact, if $f \in \mathcal{I}^{0}$, then the equation $f=0$ defines locally a submanifold $N \subset M$ and any integral manifold of $\mathcal{I}$ must be contained in $N$. Thus, instead of $\mathcal{I}$, one can consider the ideal $\left.\mathcal{I}\right|_{N}$, obtained by restricting the forms of $\mathcal{I}$ to $N$.

A distribution $\mathcal{D}=\left\{\mathcal{D}_{x} \subset T_{x} M: x \in M\right\}$ on $M$ is called a smooth distribution of dimension $r$ if and only if for each $x \in M$ there is a neighborhood $U$ of $x$ and there are $r$ smooth vector fields $Y_{1}, \ldots, Y_{r}$ which span $\mathcal{D}$ at any point of $U$. Further on we will consider only smooth distribution of constant dimension. A vector field $Y$ is said to belong to $\mathcal{D}$, i.e., $Y \in \mathcal{D}$, if and only if $Y_{x} \in \mathcal{D}_{x}$ for any $x \in M$. An integral manifold of $\mathcal{D}$ is a submanifold $i: N \rightarrow M$ such that $i_{*}\left(T_{a} N\right) \subset \mathcal{D}_{i(a)}$ for all $a \in N$. In other words, any vector field tangent to $N$ belongs to $\mathcal{D}$. A first integral of $\mathcal{D}$ is a function which is constant along the orbits of vector fields belonging to $\mathcal{D}$.

Given an exterior ideal $\mathcal{I}$, we associate to any point $x \in M$ the subspace $\operatorname{Char}_{x}(\mathcal{I}) \subset T_{x} M$ defined by

$$
\operatorname{Char}_{x}(\mathcal{I}):=\left\{\xi \in T_{x} M: \xi \sqcup \mathcal{I}_{x} \subset \mathcal{I}_{x}\right\}
$$


where $\downarrow$ denotes the interior product, and we call the distribution $\operatorname{Char}(\mathcal{I})=$ $\left\{\operatorname{Char}_{x}(\mathcal{I}), x \in M\right\}$ the characteristic distribution of $\mathcal{I}$. If $\operatorname{Char}(\mathcal{I})$ is a smooth distribution of dimension $r$, by definition, $\mathcal{I}$ is said to be nonsingular. In the following we will consider only nonsingular ideals. A vector field $Y \in \operatorname{Char}(\mathcal{I})$ is said to be a characteristic field for $\mathcal{I}$.

Note that, any smooth $r$-dimensional distribution $\mathcal{D}$ coincides with the characteristic distribution $\operatorname{Char}\left(\mathcal{I}_{\mathcal{D}}\right)$ of the exterior ideal $\mathcal{I}_{\mathcal{D}}$ generated by the $n-r$ independent 1-forms $\omega^{i}$ annihilating all the vector fields belonging to $\mathcal{D}$. Hence, the integral manifolds of $\mathcal{D}$ coincide with those of $\mathcal{I}_{\mathcal{D}}$.

An $r$-dimensional distribution $\mathcal{D}$ on $M$ is said to be completely integrable if through each point $x \in M$ passes an $r$-dimensional integral manifold. Hence, in this case, integral manifolds provide a local foliation on $M$.

One has the following

Proposition 1. If $\mathcal{I}$ is a nonsingular differential ideal, then $C h a r(\mathcal{I})$ is completely integrable.

In particular, for a simply generated exterior ideal $\mathcal{I}$, Frobenius theorem states that $C h a r(\mathcal{I})$ is completely integrable if and only if $\mathcal{I}$ is differentially closed.

For later use, let us consider the notion of infinitesimal symmetry for a distribution $\mathcal{D}$ and an exterior ideal $\mathcal{I}$.

Definition 1. A vector field $X$ on $M$ is called an infinitesimal symmetry of the distribution $\mathcal{D}$ if and only if $\mathcal{L}_{X} \mathcal{D} \subset \mathcal{D}$, i.e. $\mathcal{L}_{X} Y \in \mathcal{D}$ for any $Y \in \mathcal{D}$.

Definition 2. A vector field $X$ on $M$ is called an infinitesimal symmetry of the exterior ideal $\mathcal{I}$ if and only if $\mathcal{L}_{X} \mathcal{I} \subset \mathcal{I}$, i.e. $\mathcal{L}_{X} \theta \in \mathcal{I}$ for any $\theta \in \mathcal{I}$.

In the sequel infinitesimal symmetries of $\mathcal{D}$ or $\mathcal{I}$ will be referred to simply as symmetries. Given an exterior ideal $\mathcal{I}$, it is easy to check that any symmetry $X$ of $\mathcal{I}$ is also a symmetry of the distribution $\operatorname{Char}(\mathcal{I})$.

In order to give some applications of our results to the problem of determining first integrals of differential equations, we also recall here some basic definitions from the geometric approach to differential equations in the language of jet bundles.

Let $\pi: E \rightarrow M$ be a smooth fiber bundle with $\operatorname{dim} M=n$, fiber dimension $m$, and local coordinates $\left(x_{i}, u^{a}\right), 1 \leq i \leq n, 1 \leq a \leq m$. We denote by $J^{k}(\pi)$ the $k$ th order jet bundle with induced natural coordinates $\left(x_{i}, u_{\sigma}^{a}\right)$, where $\sigma=$ $\left(\sigma_{1}, \sigma_{2}, \ldots, \sigma_{n}\right)$ is a multi-index with $|\sigma|=\sum \sigma_{i} \leq k$ and $0 \leq \sigma_{i} \leq n$. We recall that $J^{k}(\pi)$ is equipped with the exterior ideal $\mathcal{C}$ generated by the contact forms

$$
\theta_{\sigma}^{a}=d u_{\sigma}^{a}-\sum_{i=1}^{n} u_{\sigma+1_{i}}^{a} d x^{i},
$$

where $\sigma+1_{i}:=\left(\sigma_{1}, \sigma_{2}, \ldots, \sigma_{i}+1, \ldots, \sigma_{n}\right)$. The ideal $\mathcal{C}$ is called the contact ideal and a section $s_{k}$ of $J^{k}(\pi)$ is the $k$-order prolongation of a section $s$ of $\pi$ if and only if $s_{k}^{*}\left(\theta_{\sigma}^{a}\right)=0$ for any $a=1, \ldots, m$ and $|\sigma| \leq k$. 
In this framework a $k$ th order system of differential equations can be regarded as a submanifold $\mathcal{E} \subset J^{k}(\pi)$ and any solution of $\mathcal{E}$ is a section of $\pi$ whose $k$-order prolongation is an integral manifold of $\overline{\mathcal{C}}=\left\langle\bar{\theta}_{\sigma}^{a}\right\rangle$, where $\bar{\theta}_{\sigma}^{a}$ denotes the contact forms restricted to $\mathcal{E}$. A symmetry of the contact ideal $\mathcal{C}$ which is tangent to $\mathcal{E}$ is called, by definition, a symmetry of $\mathcal{E}$.

Since contact forms are 1-forms, integral manifolds of $\overline{\mathcal{C}}$ coincide with those of $\operatorname{Char}(\overline{\mathcal{C}})$. Hence solutions of $\mathcal{E}$ can also be regarded as sections of $\pi$ whose $k$-order prolongations are integral manifolds of $\operatorname{Char}(\overline{\mathcal{C}})$. First integrals of $\operatorname{Char}(\overline{\mathcal{C}})$ will be also called first integrals of $\mathcal{E}$.

\section{Main Results}

In this section we present our main results; these relate, under suitable conditions, symmetries of an exterior ideal $\mathcal{I}$ to first integrals of $\operatorname{Char}(\mathcal{I})$. A key role will be played by condition (1) of Lemma 1 below; we also discuss relations between this condition and differential closure of $\mathcal{I}$.

Lemma 1. Let $\mathcal{I}$ be an exterior ideal of $\Lambda^{*}(M)$ generated by $\theta^{1}, \ldots, \theta^{k}$ such that

(i) if $k>1, \theta^{i} \wedge \theta^{i}=0$ for any $i=1, \ldots, k$;

(ii) $\theta^{1} \wedge \theta^{2} \wedge \cdots \wedge \theta^{k} \neq 0$ and

$$
d\left(\theta^{1} \wedge \theta^{2} \wedge \cdots \wedge \theta^{k}\right)=d K \wedge \theta^{1} \wedge \theta^{2} \wedge \cdots \wedge \theta^{k}
$$

for some $K \in C^{\infty}(M)$.

Then, for any symmetry $X$ of $\mathcal{I}$ there exists a function $K_{0} \in C^{\infty}(M)$ such that

$$
\mathcal{L}_{X}\left(\theta^{1} \wedge \theta^{2} \wedge \cdots \wedge \theta^{k}\right)=K_{0} \theta^{1} \wedge \theta^{2} \wedge \cdots \wedge \theta^{k} .
$$

Moreover, the function $G=K_{0}-X(K)$ satisfies

$$
d G \wedge \theta^{1} \wedge \theta^{2} \wedge \cdots \wedge \theta^{k}=0
$$

Proof. Since the Lie derivative is a derivation of $\Lambda^{*}(M)$ one gets

$$
\begin{aligned}
\mathcal{L}_{X}\left(\theta^{1} \wedge \theta^{2} \wedge \cdots \wedge \theta^{k}\right)= & \mathcal{L}_{X} \theta^{1} \wedge \theta^{2} \wedge \cdots \wedge \theta^{k}+\theta^{1} \wedge \mathcal{L}_{X} \theta^{2} \wedge \cdots \wedge \theta^{k} \\
& +\cdots+\theta^{1} \wedge \theta^{2} \wedge \cdots \wedge \mathcal{L}_{X} \theta^{k}
\end{aligned}
$$

On the other hand, $X$ is a symmetry of $\mathcal{I}$, i.e. for any $i=1, \ldots, k$

$$
\mathcal{L}_{X} \theta^{i}=\alpha^{i} \theta^{i}+\eta^{i}
$$

with some $\alpha^{i} \in C^{\infty}(M)$ and $\eta^{i}$ belonging to the ideal $\mathcal{I}_{(i)}=\left\langle\theta^{s}: s \neq i\right\rangle \subset \mathcal{I}$.

Therefore, by plugging (5) into (4) and taking into account assumption (i) on $\theta^{1}, \ldots, \theta^{k}$, one gets

$$
\mathcal{L}_{X}\left(\theta^{1} \wedge \theta^{2} \wedge \cdots \wedge \theta^{k}\right)=\left(\sum_{s=1}^{k} \alpha^{s}\right) \theta^{1} \wedge \theta^{2} \wedge \cdots \wedge \theta^{k} .
$$


Hence, by setting $K_{0}:=\sum_{s=1}^{k} \alpha^{s}$ one gets (2). Moreover, since $\mathcal{L}_{X} \circ d=d \circ \mathcal{L}_{X}$, assumption (ii) on $\theta^{1}, \ldots, \theta^{k}$ and (2) give (3).

Note that condition (1) is equivalent to the existence of a function $K$ such that $d\left(e^{-K} \theta^{1} \wedge \theta^{2} \wedge \cdots \wedge \theta^{k}\right)=0$. Function $K$ depends on the choice of the generators $\theta^{1}, \ldots, \theta^{k}$ of $\mathcal{I}$, however one can easily verify that conditions (i)-(ii) and function $G=K_{0}-X(K)$ are invariant under a change of generators.

Remark 1. Whenever $\mathcal{I}$ is simply generated, Eq. (3) is equivalent to $d G \in \mathcal{I}$. In fact, if the differential forms $\theta^{1}, \ldots, \theta^{k}$ are all of degree 1 , the independence Lemma (see [6] or [4]) guarantees that $d G \wedge \theta^{1} \wedge \theta^{2} \wedge \cdots \wedge \theta^{k}=0$ if and only if $d G$ is linearly dependent from $\theta^{1}, \ldots, \theta^{k}$. More in general, for any exterior ideal $\mathcal{I}$ satisfying (i), $d G \in \mathcal{I}$ implies (3) but generically the converse is not true (see for example the ideal $\mathcal{I}=\langle d x, d y \wedge d z\rangle$ and $d G=d y+d z)$. Moreover, $d G$ never belongs to $\mathcal{I}$ if none of the forms $\theta^{1}, \ldots, \theta^{k}$ has degree 1 .

For a simply generated ideal $\mathcal{I}$, the assumptions of Lemma 1 are satisfied if and only if $\theta^{1}, \ldots, \theta^{k}$ are linearly independent and $d\left(\theta^{1} \wedge \theta^{2} \wedge \cdots \wedge \theta^{k}\right)=d K \wedge \theta^{1} \wedge$ $\theta^{2} \wedge \cdots \wedge \theta^{k}$. Therefore, if $\left\{\theta^{1}, \ldots, \theta^{k}\right\}$ is completed to a coframe by adding some 1 -forms $\tau^{1}, \ldots, \tau^{h}$, for any $s=1, \ldots, k$ one has

$$
d \theta^{s}=\sum A_{i j}^{s} \tau^{i} \wedge \tau^{j}+\xi^{s}
$$

with some $A_{i j}^{s} \in C^{\infty}(M)$ and $\xi^{s} \in \mathcal{I}$. On the other hand

$$
0=d\left(\theta^{s} \wedge\left(\theta^{1} \wedge \theta^{2} \wedge \cdots \wedge \theta^{k}\right)\right)=d \theta^{s} \wedge \theta^{1} \wedge \theta^{2} \wedge \cdots \wedge \theta^{k}
$$

and by using (6) and linear independence of $\tau^{i} \wedge \tau^{j} \wedge \theta^{1} \wedge \theta^{2} \wedge \cdots \wedge \theta^{k}, i, j=1, \ldots, h$, one readily gets $A_{i j}^{s}=0$ and $d \theta^{s}=\xi^{s}$.

Hence, any simply generated ideal $\mathcal{I}$ satisfying the assumptions of Lemma 1 is differentially closed. Nevertheless, in general condition (ii) of Lemma 1 is not equivalent to the closure of $\mathcal{I}$ : just consider the ideal $\mathcal{J}=\left\langle\theta^{1}=x d u+t d y, \theta^{2}=\right.$ $d x \wedge d y \wedge d t\rangle$ (satisfying (ii) but not differentially closed) and the ideal $\mathcal{K}=\langle\theta=$ $\left.\left(y^{2} / 2\right) d x+d y\right\rangle$ (differentially closed but not satisfying (ii)).

In the particular case of an ideal generated by a 1-form $\theta$ and its differential $d \theta$, Lemma 1 can also be proved under weaker assumptions.

Lemma 2. Let $\mathcal{I}$ be an exterior ideal of $\Lambda^{*}(M)$ generated by a 1-form $\theta$ and its differential $d \theta$ such that $\theta \wedge d \theta \neq 0$ and

$$
d(\theta \wedge d \theta)=d K \wedge \theta \wedge d \theta
$$

for some $K \in C^{\infty}(M)$. For any symmetry $X$ of $\mathcal{I}$ there exists a function $K_{0} \in$ $C^{\infty}(M)$ such that

$$
\mathcal{L}_{X}(\theta \wedge d \theta)=K_{0} \theta \wedge d \theta
$$

Moreover, function $G=K_{0}-X(K)$ satisfies

$$
d G \wedge \theta \wedge d \theta=0 .
$$


Proof. Analogous to that of Lemma 1.

Now we prove the following:

Theorem 1. Let $\mathcal{I}=\left\langle\theta^{1}, \ldots, \theta^{k}\right\rangle$ be as in Lemma 1 (or Lemma 2) and $X$ a symmetry of $\mathcal{I}$. Then, function $G$ associated to $X$ by Lemma 1 (or Lemma 2) is a first integral of $\operatorname{Char}(\mathcal{I})$, i.e., is such that $Y(G)=0$ for any $Y \in \operatorname{Char}(\mathcal{I})$.

Proof. In view of (3),

$$
\begin{aligned}
0= & Y\lrcorner\left(d G \wedge \theta^{1} \wedge \theta^{2} \wedge \cdots \wedge \theta^{k}\right) \\
= & \left.Y(G) \theta^{1} \wedge \theta^{2} \wedge \cdots \wedge \theta^{k}-d G \wedge(Y\lrcorner \theta^{1}\right) \wedge \theta^{2} \wedge \cdots \wedge \theta^{k} \\
& \left.-(-1)^{\delta_{1}} d G \wedge \theta^{1} \wedge(Y\lrcorner \theta^{2}\right) \wedge \cdots \wedge \theta^{k}-\cdots \\
& \left.-(-1)^{\delta_{1}+\delta_{2}+\cdots+\delta_{k}} d G \wedge \theta^{1} \wedge \theta^{2} \wedge \cdots \wedge(Y\lrcorner \theta^{k}\right),
\end{aligned}
$$

where $\delta_{i}$ is the degree of $\theta^{i}$. Moreover, for any $i \in\{1, \ldots, k\}$, let us denote by $J_{i}$ and $I_{i}$, respectively, the (possibly void) subsets of $\{1, \ldots, k\}$ such that for $j \in J_{i}$ (respectively $j \in I_{i}$ ), $\theta^{j}$ has degree equal to (respectively less than) $\delta_{i}-1$.

Therefore, since $Y\lrcorner \mathcal{I} \subset \mathcal{I}$, one has

$$
Y\lrcorner \theta^{i}=\sum_{j \in J_{i}} b_{j}^{i} \theta^{j}+\sum_{j \in I_{i}} \beta_{j}^{i} \wedge \theta^{j}
$$

with some (possibly null) $b_{j}^{i} \in C^{\infty}(M), \beta_{j}^{i} \in \Lambda^{*}(M)$.

Then, by plugging (10) into (9) and recalling that $\theta^{s} \wedge \theta^{s}=0$, (9) reduces to

$$
0=Y\lrcorner\left(d G \wedge \theta^{1} \wedge \theta^{2} \wedge \cdots \wedge \theta^{k}\right)=Y(G)\left(\theta^{1} \wedge \theta^{2} \wedge \cdots \wedge \theta^{k}\right) .
$$

Now, since $\theta^{1} \wedge \theta^{2} \wedge \cdots \wedge \theta^{k} \neq 0$, the thesis readily follows.

Remark 2. Condition (1) of Lemma 1 can be weakened by requiring that $d\left(\theta^{1} \wedge\right.$ $\left.\theta^{2} \wedge \cdots \wedge \theta^{k}\right)=\alpha \wedge \theta^{1} \wedge \theta^{2} \wedge \cdots \wedge \theta^{k}$ for a 1-form $\alpha$ such that $\left.Y\right\lrcorner d \alpha=0$ for any $Y \in \operatorname{Char}(\mathcal{I})$. In this case we can find the first integral $\left.G=K_{0}-Y\right\lrcorner \alpha$. An analogous result holds also for condition (8) of Lemma 2.

\section{Applications to Distributions and Differential Equations}

In this section, we provide some applications of Theorem 1 to distributions of vector fields and differential equations.

\subsection{Completely integrable distributions}

Let $\mathcal{D}$ be a $k$-dimensional distribution on an $n$-dimensional manifold $M$. A first application of Theorem 1 to distributions can be given when the exterior ideal $\mathcal{I}_{\mathcal{D}}$, generated by the 1 -forms annihilating any vector field of $\mathcal{D}$, satisfies condition (ii) of Lemma 1. This requirement on $\mathcal{I}_{\mathcal{D}}$ is independent on the choice of the system of generators $\theta^{1}, \ldots, \theta^{n-k}$. 
One has the following:

Theorem 2. Let $\mathcal{D}$ be a $k$-dimensional distribution on a manifold $M$. If $\mathcal{I}_{\mathcal{D}}$ satisfies condition (ii) of Lemma 1 , then $\mathcal{D}$ is completely integrable and to any symmetry of $\mathcal{D}$ corresponds the first integral $G$ of $\mathcal{D}$ (defined in Theorem 1$)$.

Proof. By assumption $\mathcal{I}_{\mathcal{D}}=\left\langle\theta^{1}, \ldots, \theta^{n-k}\right\rangle$ satisfies condition (ii) of Lemma 1. Then $\mathcal{I}_{\mathcal{D}}=\left\langle\theta^{1}, \ldots, \theta^{n-k}\right\rangle$ is differentially closed and, by Proposition $1, \mathcal{D}$ is completely integrable. Moreover, in this case, any symmetry of $\mathcal{D}$ is also a symmetry of $\mathcal{I}_{\mathcal{D}}$. Therefore, by Theorem 1 , to any symmetry of $\mathcal{D}$ corresponds a first integral of $\mathcal{D}$.

Another application of Theorem 1 to distributions can be given when $M$ is an orientable manifold with a volume form $\Omega$.

In this case, if we consider a system $Y_{1}, \ldots, Y_{k}$ of vector fields generating $\mathcal{D}$, we apply Theorem 1 to the exterior ideal $\mathcal{I}$ generated by the $(n-k)$-form $\omega$ defined as

$$
\left.\left.\omega=Y_{1}\right\lrcorner \cdots-Y_{k}\right\lrcorner \Omega \text {. }
$$

Clearly, $\omega$ depends on the choice of $Y_{1}, \ldots, Y_{k}$, but we still note that condition (ii) of Lemma 1 is independent from the particular choice of $Y_{1}, \ldots, Y_{k}$. Moreover, by nondegeneracy of $\Omega$, it is easy to check that $\mathcal{D}=\operatorname{Char}(\mathcal{I})$.

Now, we give the following:

Theorem 3. Let $M$ be an orientable manifold with a volume form $\Omega$ and $\mathcal{D}$ be a $k$-dimensional distribution on $M$ generated by vector fields $Y_{1}, \ldots, Y_{k}$. If the form $\omega$ defined by (11) satisfies condition (ii) of Lemma 1 , then $\mathcal{D}$ is completely integrable and to any symmetry of $\mathcal{D}$ corresponds a first integral $G$ of $\mathcal{D}$.

Proof. By assumption, one has that $d \omega=d K \wedge \omega$ for some function $K$. Therefore, $\mathcal{I}$ is a differential ideal and in view of Proposition 1, the distribution $\mathcal{D}=\operatorname{Char}(\mathcal{I})$ is completely integrable. Moreover, any symmetry of $\mathcal{D}$ is also a symmetry of $\mathcal{I}$. In fact, if $X$ is a symmetry of $\mathcal{D}$ there exist some functions $a_{i}^{j} \in C^{\infty}(M)$ such that $\mathcal{L}_{X} Y_{i}=\sum a_{i}^{j} Y_{j}$ and

$$
\begin{aligned}
\mathcal{L}_{X} \omega= & \left.\left.\left.\left.\left.\left.\mathcal{L}_{X}\left(Y_{1}\right\lrcorner \cdots\right\lrcorner Y_{k}\right\lrcorner \Omega\right)=\mathcal{L}_{X} Y_{1}\right\lrcorner \cdots\right\lrcorner Y_{k}\right\lrcorner \Omega+\cdots \\
& \left.\left.\left.\left.\left.\left.Y_{1}\right\lrcorner \cdots\right\lrcorner \mathcal{L}_{X} Y_{k}\right\lrcorner \Omega+Y_{1}\right\lrcorner \cdots\right\lrcorner Y_{k}\right\lrcorner \mathcal{L}_{X} \Omega \\
= & \left(\sum_{i} a_{i}^{i}+\operatorname{div} X\right) \omega,
\end{aligned}
$$

where we have used $\left.\left.Y_{i}\right\lrcorner Y_{i}\right\lrcorner \Omega=0$ and $\mathcal{L}_{X} \Omega=(\operatorname{div} X) \Omega$ as $\Omega$ is a volume form. Hence, symmetries of $\mathcal{I}$ and of $\operatorname{Char}(\mathcal{I})$ coincide. Finally, by Lemma 1, the first integral $G$ of $\mathcal{D}$ corresponding to a symmetry $X$ has the form $G=\sum_{i} a_{i}^{i}+\operatorname{div} X-$ $X(K)$. 
Remark 3. Consider the 1-dimensional distribution $\mathcal{D}$ generated by a single vector field $Y$ on an orientable manifold $M$. If $\operatorname{div} Y=0$, then one has $d \omega=0$. In this sense Theorem 3 extends a result of Crampin (see [7]) associating to any symmetry $X$ of a divergenceless vector field $Y$ a first integral of $Y$. In this case, if $\mathcal{L}_{X} Y=a Y$ we find $G=a+\operatorname{div} X$.

When $M$ is orientable, one can prove that $\mathcal{I}_{\mathcal{D}}$ satisfies assumption (ii) of Lemma 1 if and only if $\mathcal{I}=\langle\omega\rangle$ does. Moreover, Theorems 2 and 3 associate the same first integral to any given symmetry $Y$ of $\mathcal{D}$. In fact, given $\mathcal{I}_{\mathcal{D}}=\left\langle\theta^{1}, \ldots, \theta^{n-k}\right\rangle$, by extending $\left\{\theta^{1}, \ldots, \theta^{n-k}\right\}$ to a co-frame $\left\langle\theta^{1}, \ldots, \theta^{n-k}, \tau^{1}, \ldots, \tau^{k}\right\rangle$ one can write $\Omega$ as $\Omega=\tau^{1} \wedge \cdots \wedge \tau^{k} \wedge \theta^{1} \wedge \cdots \wedge \theta^{n-k}$. Hence $\omega$ can be written as $\omega=f \theta^{1} \wedge \cdots \wedge \theta^{n-k}$ for some $f \in C^{\infty}(M)$. It readily follows that $\mathcal{I}_{\mathcal{D}}$ satisfies assumption (ii) with $K=K_{1}$ if and only if $\mathcal{I}=\langle\omega\rangle$ does with $K=K_{1}+\ln |f|$. We have proved that any symmetry $X$ of $\mathcal{D}$ is also a symmetry of $\mathcal{I}_{\mathcal{D}}$ and $\mathcal{I}$. Hence, if $\mathcal{L}_{X}\left(\theta^{1} \wedge \theta^{2} \wedge \cdots \wedge \theta^{n-k}\right)=$ $K_{0} \theta^{1} \wedge \theta^{2} \wedge \cdots \wedge \theta^{n-k}$, by applying Theorem 1 to $\mathcal{I}_{\mathcal{D}}$ we find the first integral $G=K_{0}-X\left(K_{1}\right)$, whereas by applying Theorem 1 to $\mathcal{I}$ we find the first integral $\bar{G}=X(f) / f+K_{0}-X\left(K_{1}+\ln |f|\right)=G$.

\subsection{Ordinary differential equations}

In this subsection we give an application of Theorem 1 to systems of ODEs; in the notation above this corresponds to the choice $\mathcal{I}=\overline{\mathcal{C}}$.

Since in the case of under-determined systems $\overline{\mathcal{C}}$ cannot be differentially closed, we only consider the case of $k$-order systems of $m$ ODEs in $m$ unknown functions. Moreover, when such a system is solved with respect to higher order derivatives, a coordinate description of condition (1) of Lemma 1 will be given. Let us start with the following application of Theorem 2 :

Theorem 4. Let $\mathcal{E} \subset J^{k}(\pi)$ be a determined system of $m$ ordinary differential equations of order $k$ and $\overline{\mathcal{C}}$ the corresponding contact ideal restricted to $\mathcal{E}$. If $\overline{\mathcal{C}}$ satisfies assumption (ii) of Lemma 1 , then to any symmetry $X$ of $\overline{\mathcal{C}}$ is associated the first integral $G$ defined by formula (2).

Proof. The contact ideal $\overline{\mathcal{C}}$ satisfies the assumptions of Lemma 1 . Therefore, to any symmetry $X$ of $\overline{\mathcal{C}}$ it is associated a function $G$ which is invariant under the flow of any vector field in $\operatorname{Char}(\overline{\mathcal{C}})$. Hence $G$ is constant along integral manifolds of $\operatorname{Char}(\overline{\mathcal{C}})$ and in particular along any solution of $\mathcal{E}$.

For the sake of completeness we also provide a coordinate description of assumption (1) when $\mathcal{I}=\overline{\mathcal{C}}$ and $\mathcal{E}$ can be solved with respect to higher derivatives.

Since any $k$-order system of ODEs $\mathcal{E} \subset J^{k}(\pi)$ can always be reduced to a first order one, we consider only this case. In local coordinates $\left(t, u^{a}, u_{t}^{a}\right)$, a first-order 
system in normal form reads

$$
\left\{\begin{array}{l}
u_{t}^{1}=f^{1}\left(t, u^{a}, u_{t}^{a}\right), \\
\vdots \\
u_{t}^{m}=f^{m}\left(t, u^{a}, u_{t}^{a}\right) .
\end{array}\right.
$$

for some functions $f^{1}, \ldots, f^{m}$, with $a=1, \ldots, m$. Thus, the contact forms restricted to $\mathcal{E}$ are

$$
\bar{\theta}^{a}=d u^{a}-f^{a} d t
$$

and a straightforward computation shows that condition (1) of Lemma 1 reads

$$
\frac{\partial K}{\partial t}+f^{1} \frac{\partial K}{\partial u^{1}}+\cdots+f^{m} \frac{\partial K}{\partial u^{m}}=\frac{\partial f^{1}}{\partial u^{1}}+\frac{\partial f^{2}}{\partial u^{2}}+\cdots+\frac{\partial f^{m}}{\partial u^{m}} .
$$

Note that the left-hand side of (13) is the total derivative of function $K$ restricted to $\mathcal{E}$.

\subsection{First-order PDE}

In this subsection we present an application of Theorem 1 to first-order PDEs. Given a first-order PDE $\mathcal{E}=\left\{F\left(x^{i}, u, u_{x^{i}}\right)=0, i=1, \ldots, n\right\}$ we consider the exterior ideal $\mathcal{I}=\langle\bar{\theta}, d \bar{\theta}\rangle$ generated by the restricted contact form $\bar{\theta}$ and its differential $d \bar{\theta}$. Theorem 5 below associates to any symmetry of $\mathcal{I}$ a first integral of $\mathcal{E}$, i.e., a function which is constant along any characteristic vector field of $\mathcal{I}$. Note that, by means of the Lagrange-Charpit method (see [19,20]), one can use first integrals to construct complete integrals of $\mathcal{E}$. Now, we have the following

Theorem 5. Given a first order PDE $\mathcal{E}$, if $\mathcal{I}=\langle\bar{\theta}, d \bar{\theta}\rangle$ satisfies assumptions of Lemma 2 , then to any symmetry $X$ of $\mathcal{I}$ is associated a first integral of $\mathcal{E}$.

Proof. This is a straightforward consequence of Theorem 1 since the assumptions of Lemma 2 are satisfied and $\operatorname{Char}(\mathcal{I})$ is one-dimensional.

Remark 4. The ideal $\mathcal{I}=\langle\bar{\theta}, d \bar{\theta}\rangle$ satisfies the assumptions of Lemma 2 with $K=$ constant iff $F_{u}=0$ and $n=2$, i.e., $\mathcal{E}$ is an Hamilton-Jacobi type equation with only two independent variables. On the other hand, as observed in Remark 2, Theorem 5 can be applied to a wider class of PDEs (see Example 4).

\section{Examples}

In this section we collect some simple examples, related to Theorems 3,4 , and 5 , in order to show that the considered class of exterior ideal $\mathcal{I}$ is not void. 
Example 1. Consider the distribution on $\mathbb{R}^{3}-\{x=0\}$ generated by the vector fields

$$
\begin{aligned}
& Y_{1}=\left(1-e^{x}\right) \partial_{x}+y\left(e^{x}+1\right) \partial_{y}+\left(2 y-2 y e^{x}-z\right) \partial_{z}, \\
& Y_{2}=\frac{e^{x}}{\left(1-e^{x}\right)^{2}} \partial_{y}+\frac{e^{x}}{1-e^{x}} \partial_{z}
\end{aligned}
$$

and the volume form $\Omega=d x \wedge d y \wedge d z$. In this case $\left.\theta=Y_{1} \dashv Y_{2}\right\lrcorner \Omega$ is closed and the vector field

$$
X=\frac{\left(z-y+y e^{x}\right) e^{x}}{2} \partial_{x}+\frac{y\left(z-y+y e^{x}\right) e^{2 x}}{2\left(1-e^{x}\right)} \partial_{y}
$$

is a symmetry of $\theta$ such that

$$
\mathcal{L}_{X}(\theta)=e^{x}\left(\frac{z-y+y e^{x}}{e^{x}-1}\right) \theta .
$$

Therefore, one gets the joint invariant $G=e^{x}\left(\frac{z-y+y e^{x}}{e^{x}-1}\right)$ for $Y_{1}, Y_{2}$.

Example 2. Consider the Hamiltonian system $\mathcal{E} \subset J^{1}(\pi)$, with $\pi: \mathbb{R}^{5} \rightarrow \mathbb{R}$, defined by

$$
\begin{aligned}
& \dot{q}_{1}=p_{1}, \\
& \dot{q}_{2}=p_{2}, \\
& \dot{p}_{1}=-q_{1}, \\
& \dot{p}_{2}=-q_{2},
\end{aligned}
$$

with $\left(q_{1}, q_{2}, p_{1}, p_{2}\right)$ fiber coordinates and $t$ coordinate on the base $\mathbb{R}$. It is easy to check that (14) satisfy (13). Moreover, the vector field

$$
\begin{aligned}
X= & q_{1} \sin t \partial_{t}+q_{1}^{2} \cos t \partial_{q_{1}}+q_{1} q_{2} \cos t \partial_{q_{2}}+\left(q_{1} p_{1} \cos t-q_{1}^{2} \sin t-p_{1}^{2} \sin t\right) \partial_{p_{1}} \\
& +\left(q_{2} p_{1} \cos t-p_{1} p_{2} \sin t-q_{1} q_{2} \sin t\right) \partial_{p_{2}}
\end{aligned}
$$

is a symmetry of the ideal $\overline{\mathcal{C}}$ generated by

$$
\bar{\theta}^{1}=d q_{1}-p_{1} d t, \quad \bar{\theta}^{2}=d q_{2}-p_{2} d t, \quad \bar{\theta}^{3}=d p_{1}+q_{1} d t, \quad \bar{\theta}^{4}=d p_{2}+q_{2} d t .
$$

Then, one gets that

$$
\mathcal{L}_{X}\left(\bar{\theta}^{1} \wedge \bar{\theta}^{2} \wedge \bar{\theta}^{3} \wedge \bar{\theta}^{4}\right)=4\left(q_{1} \cos t-p_{1} \sin t\right) \bar{\theta}^{1} \wedge \bar{\theta}^{2} \wedge \bar{\theta}^{3} \wedge \bar{\theta}^{4}
$$

i.e. $G=4\left(q_{1} \cos t-p_{1} \sin t\right)$.

Example 3. Consider the equation $\mathcal{E}=\left\{F\left(x, y, u_{x}, u_{y}\right)=0\right\}$ with

$$
F:=h\left(y, \frac{u_{y}}{u_{x}}\right)-\frac{1}{u_{x}}+x,
$$

where $h\left(y, u_{y} / u_{x}\right)$ is an arbitrary function. The differential ideal $\mathcal{I}$ is generated by $\bar{\theta}$ and $d \bar{\theta}$, with

$$
\bar{\theta}=d u+u_{x}\left(d h+\frac{1}{u_{x}^{2}} d u_{x}\right)-u_{y} d y
$$


It is easy to check that the vector field

$$
X=e^{u}\left(\partial_{x}-u_{x}^{2} \partial_{u_{x}}-u_{x} u_{y} \partial_{u_{y}}\right)
$$

is a symmetry of $\mathcal{I}$, and

$$
\mathcal{L}_{X}(\bar{\theta} \wedge d \bar{\theta})=-u_{x} e^{u}(\bar{\theta} \wedge d \bar{\theta})
$$

i.e. $G=u_{x} e^{u}$ is a first integral of $\mathcal{E}$.

Example 4. Consider the nonlinear wave equation $\mathcal{E}=\left\{F\left(x, y, u, u_{x}, u_{y}\right)=0\right\}$ with

$$
F:=u_{x}-u u_{y} .
$$

The differential ideal $\mathcal{I}$, generated by $\bar{\theta}$ and $d \bar{\theta}$ with

$$
\bar{\theta}=d u-u u_{y} d x-u_{y} d y
$$

satisfies condition $d(\bar{\theta} \wedge d \bar{\theta})=\alpha \wedge \bar{\theta} \wedge d \bar{\theta}$ with, for example, $\alpha=d x+(1 / u) d y+$ $(x / u) d u+\left(2 / u_{y}\right) d u_{y}$. In this case the characteristic vector field is $Y=\partial_{x}-u \partial_{y}+$ $u_{y}^{2} \partial_{u_{y}}$ and $\left.Y\right\lrcorner d \alpha=0$. Hence (see Remark 2) to any symmetry $X$ of $\mathcal{I}$ one can associate the first integral $G=K_{0}-X \sqcup \alpha$. In particular, if we consider the symmetry (see [14])

$$
X=y \partial_{x}+u^{2} \partial_{u}+u u_{y} \partial_{u_{y}},
$$

we get the first integral $G=2 u+u x+y$.

\section{Acknowledgment}

The authors would like to thank Giuseppe Gaeta for his comments and suggestions. This work was partially supported by GNFM-INDAM through the project 'Simmetrie e riduzione per PDE, principi di sovrapposizione e structture nonlocali'.

\section{Appendix A. ODEs of Variational Type}

In this appendix, we apply the results of Lemma 1 to a system $\mathcal{E}$ of ordinary Euler-Lagrange equations. In this case, we associate to $\mathcal{E}$ an exterior ideal which, exploiting the variational structure of $\mathcal{E}$, automatically satisfies condition (ii) of Lemma 1 . In fact, in this case one can consider the ideal $\mathcal{I}$ generated by the exterior derivative of the Hamilton-Cartan form associated to the Lagrangian $L$.

We recall that in the case of a first-order variational problem on a bundle $\pi$ : $E \rightarrow N$ with $\operatorname{dim}(N)=1$, the Hamilton-Cartan form reads $\Theta=\left(\partial L / \partial \dot{q}^{i}\right)\left(d q^{i}-\right.$ $\left.\dot{q}^{i} d t\right)+L d t$, where $t$ is the base coordinate and $\left(q^{i}\right),\left(\dot{q}^{i}\right)$ are fiber coordinates and first-order jet coordinates, respectively.

Here follows an application of Theorem 1 to this case.

Theorem 6. Let $\Theta$ be the Hamilton-Cartan form for a first-order variational problem on a bundle $\pi: E \rightarrow N$, where $\operatorname{dim}(N)=1$, with a regular Lagrangian. Then 
to any symmetry of the ideal $\mathcal{I}=\langle d \Theta\rangle$ corresponds a first integral for the EulerLagrange equations of $\Theta$.

Proof. The distribution $\operatorname{Char}(\mathcal{I})$ is one-dimensional and, as the Lagrangian is regular, is generated by the vector field assigning the Euler-Lagrange equations. Therefore, since $\mathcal{I}$ satisfies the assumptions of Lemma 1, by Theorem 1 one gets the thesis.

Note that, as shown by Prince in [16], any first integral of $\mathcal{E}$ corresponds to a Cartan symmetry $X$, i.e. a field such that $\mathcal{L}_{X} d \Theta=0$. Hence, the first integral of Theorem 6 can also be obtained by using this extension of Noether theorem. However, since in our framework Cartan symmetries leads to identically zero first integrals, in order to find non-trivial first integral we have to look for non-Cartan symmetries. A limit of our approach is that not any first integral $G$ of $\mathcal{E}$ can be obtained by Theorem 6 since in that case Eq. (3) reads $d G \wedge d \Theta=0$.

\section{References}

[1] D. V. Alexseevskij, A. M. Vinogradov and V. V. Lychagin, Basic ideas and concepts of differential geometry, in Geometry I - Encyclopaedia of Mathematical Sciences, Vol. 28 ed. R. V. Gamkrelidze (Springer, 1991).

[2] I. Benn, Conservation laws for divergenceless differential equations, J. Phys. A: Math. Gen. 25 (1992) 6723-6731.

[3] G. Bluman, Connections between symmetries and conservation laws, SIGMA 1 (2005) $1-16$.

[4] R. L. Bryant, S. S. Chern, R. B. Gardner, H. L. Goldschmidt and P. A. Griffiths, Exterior Differential Systems (Springer, 1991).

[5] E. Cartan, Les systèmes différentielles extérieurs et leur applications géométriques (Hermann and Cie, 1945).

[6] S. S. Chern, W. H. Chen and K. S. Lam, Lectures on Differential Geometry (World Scientific, 2000).

[7] M. Crampin, Symmetries and invariants of volume-preserving flows, Phys. Lett. A 79 (1980) 138-140.

[8] S. V. Duzhin and V. V. Lychagin, Symmetries of distributions and quadrature of ordinary differential equations, Acta Appl. Math. 24 (1991) 29-57.

[9] G. Gaeta, P. Morando and T. O. Turgut, Symmetry reduction in the variational approach to Liouville dynamics, Int. J. Geom. Meth. Mod. Phys. 2 (2005) 657-674.

[10] F. G. Gascon, Divergence-free vectorfields and integration via quadratures, Phys. Lett. A 225 (1997) 269-273.

[11] P. A. Griffiths, Exterior Differential Systems and the Calculus of Variations (Birkhauser, 1983).

[12] S. A. Hojman, A new conservation law constructed without using either Lagrangians or Hamiltonians, J. Phys. A: Math. Gen. 25 (1992) L291-L295.

[13] G. L. Jones, Symmetry and conservation laws of differential equations, Nuovo Cimento Soc. Ital. Fis. B 112 (1997) 1053-1059.

[14] P. J. Olver, Applications of Lie Groups to Differential Equations (Springer, 1993).

[15] P. J. Olver, Equivalence, Invariants, and Symmetry (Cambridge University Press, 1995). 
[16] G. Prince, Toward a classification of dynamical symmetries in classical mechanics, Bull. Austral. Math. Soc. 27 (1983) 53-71.

[17] V. Rosenhaus and G. H. Katzin, On symmetries, conservation laws, and variational problems for partial differential equations, J. Math. Phys. 35 (1994) 1998-2012.

[18] J. Sherring and G. Prince, Geometric aspects of reduction of order, Trans. Amer. Math. Soc. 334 (1992) 433-453.

[19] V. I. Smirnov, A Course of Higher Mathematics (Pergamon Press, 1964).

[20] A. M. Vinogradov et al., Symmetries and Conservation Laws for Differential Equations of Mathematical Physics (American Mathematical Society, 1999). 